\title{
Factors Associated with Postoperative Chronic Pain and Recurrence After Laparoscopic Total Extraperitoneal Inguinal Hernia Repair
}

\author{
(D) Yaşar Özdenkaya ${ }^{1}$, (D) Naciye Çiğdem Arslan ${ }^{1}$, (D) Pelin Basım ${ }^{1}$, (D) Oktay Olmuşçelik ${ }^{2}$ \\ 1 Istanbul Medipol University Faculty of Medicine, Department of Surgery, Istanbul, Turkey \\ 2istanbul Medipol University Faculty of Medicine, Department of Internal Medicine, istanbul, Turkey
}

\section{Abstract}

Objective: To assess the risk factors for chronic pain and recurrence after laparoscopic total extraperitoneal (TEP) inguinal hernia repair.

Methods: Data of the patients who underwent laparoscopic TEP repair were analyzed. Clinical and surgical characteristics, including learning curve, mesh weight, the pore size of the mesh, use of mesh fixation, and fixation methods, were assessed. Fixation by absorbable or nonabsorbable tackers was performed in all procedures except for self-fixating meshes. Mesh brands were tiered into groups regarding pore size and weight. Operative time was defined as the duration between skin incision and dressing. The minimum follow-up was 24 months. Postoperative chronic pain was defined as moderate to severe groin pain, which was accepted as a Visual Analog score $\geq 3$ longer than three months. Pain and recurrence were evaluated at 1 and 6 weeks in clinic visits and at 3, 12, and 24 months by telephone questionnaires. The occurrence of a fascial defect in physical examination or ultrasound was defined as recurrence.

Results: Three-hundred and eighty-two procedures were included. Postoperative chronic pain was seen in 31 (8.1\%) patients and was higher with micropore mesh $(p=0.004)$, mesh fixation $(p=0.002)$, fixation with titanium tacks $(p<0.001)$ and at first 50 cases $(p=0.043)$. Fifteen $(3.9 \%)$ patients had a recurrence. Older age $(p=0.046)$, prolonged operative time $(p=0.040)$, body mass index $(B M I)(p=0.008)$ and learning curve $(p=0.034)$ were significantly associated with higher recurrence rate. In multivariate analysis pore size [Odds ratio (OR): $2.911,95 \%$ confidence interval (CI): 1.153-7.351, $\mathrm{p}=0.024$ ] and fixation with titanium tacks (OR: 8.776, 95\% Cl: 4.040-14.893, $\mathrm{p}=0.004)$ were independent risk factors for chronic pain; BMI (OR: 1.307-95\% Cl:1.138-1.501, $\mathrm{p}<0.001)$ was the only independent risk factor for recurrence.

Conclusion: The outcome of laparoscopic TEP repair is related to the technic as well as patient-based factors. Titanium tacks and micropore meshes increase postoperative pain risk without any benefit on recurrence. Patients with higher BMI have an increased recurrence risk.

Keywords: Laparoscopy, hernia repair, inguinal hernia, total extraperitoneal repair, groin hernia

\section{INTRODUCTION}

The main goal of the on inguinal hernia repair has been the better surgical outcome, particularly regarding chronic pain and recurrence during recent years. Approximately $10 \%$ of the patients suffer from chronic pain (longer than three months), and 10-15\% require reoperation for recurrence after open or laparoscopic inguinal hernia repair (1). Minimally invasive approaches that provide reduced nerve damage and postoperative pain with satisfactory recurrence rates have been emerged to improve quality of life after inguinal hernia repair (2). In a meta-analysis comparing laparoscopic transabdominal preperitoneal and total extraperitoneal (TEP) technics with Lichtenstein procedure revealed no difference in recurrence rates but fewer wound infection and chronic pain and earlier return to work with laparoscopic technics (3). Laparoscopic TEP is the most preferred minimally invasive repair with fewer complications, including visceral injury, ileus, and port-site hernia, shorter hospital stay, and reduced postoperative pain $(4,5)$. In a prospective study, 10.555 Lichtenstein and 6.833 TEP procedures were compared,

Phone: +90 5313890975 E-mail: cigdemarslan@hotmail.it ORCID ID: orcid.org/0000-0003-0428-2511

Cite this article as: Özdenkaya Y, Arslan NÇ, Basım P, Olmuşçelik O. Factors Associated with Postoperative Chronic Pain and Recurrence After Laparoscopic Total Extraperitoneal Inguinal Hernia Repair. Eur Arch Med Res 2020; 36 (1):57-62

๑Copyright 2020 by the University of Health Sciences Turkey, Okmeydanı Training and Research Hospital

European Archives of Medical Research published by Galenos Publishing House. 
and no difference in recurrence and chronic pain was found, however postoperative complications $(p<0.001)$ and early pain at rest $(p=0.011)$ and exertion $(p<0.001)$ were lower in TEP group (6).

Despite several randomized trials and meta-analyses, guidelines still do not mention any of those procedures as the standard of care (7). Either Lichtenstein or laparoscopic technics can be chosen based on resources and expertise. Moreover, there is no consensus on mesh selection and fixation (7). There is still a need for large-scale, controlled studies comparing different types of surgical materials. This study presents the mid-term results of TEP repair in a single center with an analysis of risk factors for chronic pain and recurrence.

\section{METHODS}

Prospectively collected database of patients who underwent laparoscopic TEP inguinal hernia repair between January 2012 and January 2017 was reviewed. Patients older than 18 years old with primary inguinal hernia were included in the study. All the procedures were performed under general anesthesia by the same team. The preoperative condition of the patients was recorded according to the American Society of Anesthesiologists classification. Age, gender, body mass index (BMI), type of hernia (unilateral and bilateral), type of defect (direct, indirect and combined), mesh weight, pore size of the mesh, use of mesh fixation and fixation methods (absorbable tackers and titanium tackers) were retrospectively gathered from the registry.

Mesh brands were tiered into groups regarding pore size and weight. BARD 3DmaxTM (Bard Davol Inc., RI, USA) and ProleneTM (Ethicon, LLC, USA) mesh comprised micropore group, where ProGripTM (Medtronic, MN, USA) and ParietexTM (Medtronic, $M N$, USA) meshes were included in macropore group. BARD 3DmaxTM, ProleneTM, Parietex Hydrophilic Anatomical Mesh ${ }^{T M}$ and ProGrip TM mesh were included in heavy-weight $(>50$ $\left.\mathrm{g} / \mathrm{m}^{2}\right)$ group. The light-weighted group was comprised of Parietex Lightweight MeshTM. Tacker fixation by ProtackTM or AbsorbatackTM was used in all procedures except for those performed using ProGripTM. The selection of mesh and tacker brands was due to the subjective choice of the surgeon and the availability of the materials at present.

Operative time was defined as the duration between skin incision and dressing. The procedures were divided into two groups (first 50 cases and after 50 cases) to assess the effect of the learning curve. Postoperative analgesia was provided with paracetamol and diclofenac sodium. All patients were assessed for Visual Analog scale score (VAS) and examined for seroma and recurrence at 1 and 6 weeks. At 3, 12 and 24 months, telephone questionnaire was performed, and VAS scores were recorded. Patients with any complaints were invited to the outpatient clinic for an in-person examination. Postoperative chronic pain was defined as moderate to severe groin pain, which was accepted as a VAS score lasting greater than 3 for 3 months. Recurrence was evaluated by physical examination, and ultrasound was performed for confirmation in case. Any fascial defect in the previous hernia site determined by the surgeon was recorded as recurrence. If the physical examination did not reveal a finding associated with a recurrent hernia, ultrasound was performed in patients with symptoms.

\section{Statistical Analysis}

Statistical analysis was done using IBM SPSS Statistics for Windows, Version 24.0. Categorical data were compared by chisquare or Fisher's exact tests. Continuous data were compared using the Student's t-test. A multivariate logistic regression model was conducted to determine risk factors for chronic pain and recurrence. $\mathrm{P}<0.05$ was considered statistically significant.

Approval of the local Ethics committee was received for the study (Istanbul Medipol University Ethics committee number: 10840098-604.01.01-E.53651). All the patients gave written consent for the surgery and permission for the use of data in studies.

\section{RESULTS}

Two hundred and fourteen patients were meeting the inclusion criteria. Bilateral repairs were assessed individually, and 382 procedures were analyzed. The demographic and surgical characteristics of the patients are given in Table 1. The brand of the mesh was BARD 3Dmax mesh in 64 (16.8\%) patients, Parietex Lightweight mesh in 44 (11.5\%), Parietex Hydrophilic Anatomical mesh in 77 (20.2\%), ProGrip Laparoscopic self-fixating mesh in $69(18.1 \%)$ and Prolene mesh in 128 (33.5\%) patients. There were $192(50.3 \%)$ and 190 (49.7\%) patients in micropore and macropore groups. High-weighted meshes were used in 338 (88.5\%) patients; 44 (11.5\%) patients had light-weighted mesh. No fixation was performed in 69 (18\%) patients who underwent hernia repair using self-fixating mesh.

The mean VAS score at one week was 3.6 \pm 1.9 ; at six weeks was $1.4 \pm 0.8$; at three months was $0.57 \pm 0.4$; at six months was $0.32 \pm 0.3$, at 12 months $0.22 \pm 0.5$ and $0.1 \pm 0.2$ at 24 months. Postoperative chronic pain was seen in 31 (8.1\%) patients. Conservative treatments were given to those patients. At 12 months, only 5 (1.3\%) patients had remaining chronic pain. 
The mean BMI was $26.4 \pm 3.2$ in patients with chronic pain and significantly higher than the mean BMI of patients having no pain (24.8 $\pm 2.7, p=0.010$ ). Type of the hernia or defect and mesh weight was no effect on postoperative pain. Postoperative chronic pain was seen in $23(12 \%)$ patients in the microporous mesh group and 8 (4.2\%) patients in the macroporous group $(p=0.004)$. Mesh fixation significantly increased postoperative pain rate. None of the patients with self-fixating meshes suffered chronic pain. The chronic pain rate was significantly higher with mesh fixation ( $0 \%$ vs $9.9 \%, p=0.002$ ). Among 31 patients with chronic pain, 30 had titanium tacks, and 1 had absorbable tacks.

\begin{tabular}{|c|c|}
\hline Number of patients & 382 \\
\hline Age (mean $\pm S D)$ & $50.8 \pm 14.9$ \\
\hline \multicolumn{2}{|l|}{ Sex } \\
\hline Male & $359(94 \%)$ \\
\hline Female & $23(6 \%)$ \\
\hline BMI $\left(\mathrm{kg} / \mathrm{m}^{2}\right.$, mean $\left.\pm \mathrm{SD}\right)$ & $24.9 \pm 2.8$ \\
\hline \multicolumn{2}{|l|}{ ASA class } \\
\hline $1-2$ & $303(79.3 \%)$ \\
\hline $2-4$ & $79(20.7 \%)$ \\
\hline \multicolumn{2}{|l|}{ Type of hernia } \\
\hline Unilateral & $45(11.8 \%)$ \\
\hline Bilateral & $337(88.2 \%)$ \\
\hline \multicolumn{2}{|l|}{ Type of defect } \\
\hline Direct & $146(38.2 \%)$ \\
\hline Indirect & $220(56.7 \%)$ \\
\hline Combined & $16(4.2 \%)$ \\
\hline \multicolumn{2}{|l|}{ Type of mesh } \\
\hline Highweight & $338(88.5 \%)$ \\
\hline Lightweight & $44(11.5 \%)$ \\
\hline \multicolumn{2}{|l|}{ Pore size } \\
\hline Micropore & $192(50.3 \%)$ \\
\hline Macropore & $190(49.7 \%)$ \\
\hline \multicolumn{2}{|l|}{ Mesh fixation } \\
\hline No & $69(18 \%)$ \\
\hline Yes & 313 \\
\hline Titanium & 187 (49\%) \\
\hline Absorbable & $126(33 \%)$ \\
\hline Operative time ( $\min \pm S D$ ) & $39.6 \pm 14.1$ \\
\hline \multicolumn{2}{|l|}{ Learning curve } \\
\hline First 50 cases & 50 \\
\hline After 50 cases & $332(86.9 \%)$ \\
\hline
\end{tabular}

Titanium tacks were associated with higher pain rate $(p<0.001)$ (Table 2).

The median follow-up was 26 (24-62) months. The recurrent hernia was seen in 15 (3.9\%) patients who had significantly higher mean BMI ( $27.9 \pm 3.8$ vs $24.8 \pm 2.7, p=0.008)$. Female patients had no recurrence $(p=0.387)$. The mean age was significantly higher in patients with recurrence $(51 \pm 18.4$ vs $43.2 \pm 16.1, p=0.046)$. None of the patients with unilateral hernia suffered a recurrence, where 15 recurrences out of 337 procedures (4.5\%) were seen, but the difference between patients with unilateral and bilateral

Table 2. Univariate analysis of the factors associated with pain

\begin{tabular}{|c|c|c|c|}
\hline \multirow{2}{*}{$n=382$} & \multicolumn{2}{|l|}{ Pain } & \multirow[t]{2}{*}{ p } \\
\hline & No $(n=351)$ & Yes $(n=31)$ & \\
\hline Age (mean $\pm S D)$ & $50.6 \pm 14.3$ & $52.7 \pm 19.7$ & 0.457 \\
\hline \multicolumn{3}{|l|}{ Sex } & 0.575 \\
\hline Male & 330 & $29(8.1 \%)$ & \\
\hline Female & 21 & $2(8.7 \%)$ & \\
\hline BMI $\left(\mathrm{kg} / \mathrm{m}^{2}\right.$, mean $\left.\pm \mathrm{SD}\right)$ & $24.8 \pm 2.7$ & $26.4 \pm 3.2$ & 0.010 \\
\hline \multicolumn{3}{|l|}{ ASA class } & 0.349 \\
\hline $1-2$ & 277 & $26(8.6 \%)$ & \\
\hline $2-4$ & 74 & $5(6.3 \%)$ & \\
\hline \multicolumn{3}{|l|}{ Type of hernia } & 0.264 \\
\hline Unilateral & 43 & $2(4.4 \%)$ & \\
\hline Bilateral & 308 & $29(8.6 \%)$ & \\
\hline \multicolumn{3}{|l|}{ Type of defect } & 0.895 \\
\hline Direct & 135 & $11(7.5 \%)$ & \\
\hline Indirect & 201 & $19(8.6 \%)$ & \\
\hline Combined & 15 & $1(6.3 \%)$ & \\
\hline \multicolumn{3}{|l|}{ Type of mesh } & 0.490 \\
\hline High weight & 311 & $27(8 \%)$ & \\
\hline Lightweight & 40 & $4(9.1 \%)$ & \\
\hline \multicolumn{3}{|l|}{ Pore size } & 0.004 \\
\hline Micropore & 169 & $23(12 \%)$ & \\
\hline Macropore & 182 & $8(4.2 \%)$ & \\
\hline \multicolumn{3}{|l|}{ Mesh fixation } & 0.002 \\
\hline No & 69 & 0 & \\
\hline Yes & 282 & $31(9.9 \%)$ & \\
\hline Titanium & 157 & $30(16 \%)$ & $<0.001$ \\
\hline Absorbable & 125 & $1(0.8 \%)$ & \\
\hline Operative time ( $\min \pm S D$ ) & $39.9 \pm 14.1$ & $36.4 \pm 14.2$ & 0.277 \\
\hline \multicolumn{3}{|l|}{ Learning curve } & 0.043 \\
\hline First 50 cases & 43 & $7(14 \%)$ & \\
\hline After 50 cases & 308 & $24(7.2 \%)$ & \\
\hline
\end{tabular}

SD: Standard deviation, BMI: Body mass index, ASA: American Society of Anesthesiologists classification, Min: Minute 
hernias was not statistically significant $(p=0.147)$. Alike as hernia type, the difference in recurrence rates in light-weighted $(0 \%)$ and high-weighted (4.4\%) mesh groups $(p=0.154)$ and also microporous (5.7\%) and macroporous (2.1\%) groups $(p=0.058)$ did not reach a statistical significance. The recurrence rate was similar in patients with and without mesh fixation. There were 3 (4.3\%) recurrences in the self-fixating mesh group and 12 (3.8\%) recurrences in patients who underwent titanium or absorbable tack fixation ( $p=0.074$ ). In the first 50 cases, $5(10 \%)$ recurrences were seen, which was significantly higher when compared with the following cases $(n=10,3.1 \%)(p=0.034)$ (Table 3).

Multivariate analysis was performed for factors predicting postoperative chronic pain (mesh fixation, fixation material, pore size, and BMI) and recurrence (BMI and learning curve). Using microporous meshes [Odds Ratio (OR): 2.911, 95\% confidence interval (Cl): 1.153-7.351, $\mathrm{p}=0.024]$ and fixation with titanium tacks (OR: $8.776,95 \% \mathrm{Cl}: 4.040-14.893, p=0.004$ ) were independently associated with postoperative pain. BMI was the only independent predictor of recurrence (OR: 1.307, 95\% CI: 1.138-1.501, $p<0.001$ ) in multivariate analysis (Table 4).

\section{DISCUSSION}

Postoperative chronic pain and recurrence are the major problems of inguinal hernia repair. Our results indicated the inessentiality of mesh fixation, which has already been shown before. Apart from confirming previous literature, this study revealed the predictive factors for postoperative pain and recurrence. We found an increased risk of pain with microporous mesh and fixation with titanium tacks. The only independent predictor of recurrence was BMI.

The incidence of chronic pain after inguinal hernia repair has been reported up to $30 \%(8,9)$ and decreased less than 3\% since laparoscopic technics emerged (10). This benefit may be associated with direct vision and protection of inguinal sensory nerves. In a study of 626 TEP repair published by Patel et al. (11) in 2016, significant and severe pain incidences were reported as $25 \%$ and $0.09 \%$ at the end of 2 years. They used Surgical Outcomes Measurement system and Carolinas Comfort scale and defined significant pain as mild but bothersome or worse on either specific tool. Mesh brand (Parietex anatomic, parietexprogrip, and physiomesh), size of the mesh, and fixation method had no effect on pain. Poor quality of life and general health status were predictors of pain longer than six months. In another study, Westin et al. (9) reported that $20 \%$ of the patients suffered from some degree of pain after TEP at one year, but severe pain was reported by $2.1 \%$. The Inguinal Pain
Questionnaire was used in this study. In our study, the VAS score $>3$ lasting longer than three months was accepted as chronic

Table 3. Univariate analysis of the factors associated with recurrence

\begin{tabular}{|c|c|c|c|}
\hline \multirow[b]{2}{*}{$n=382$} & \multicolumn{2}{|l|}{ Recurrence } & \multirow[t]{2}{*}{$\mathrm{p}$} \\
\hline & No $(n=367)$ & Yes $(n=15)$ & \\
\hline Age (mean \pm SD) & $51 \pm 18.4$ & $43.2 \pm 16.1$ & 0.046 \\
\hline \multicolumn{3}{|l|}{ Sex } & 0.387 \\
\hline Male & 344 & $15(4.2 \%)$ & \\
\hline Female & 23 & 0 & \\
\hline BMI $\left(\mathrm{kg} / \mathrm{m}^{2}\right.$, mean $\left.\pm \mathrm{SD}\right)$ & $24.8 \pm 2.7$ & $27.9 \pm 3.8$ & 0.008 \\
\hline \multicolumn{3}{|l|}{ ASA class } & 0.623 \\
\hline $1-2$ & 291 & $12(4 \%)$ & \\
\hline $2-4$ & 76 & $3(3.8 \%)$ & \\
\hline \multicolumn{3}{|l|}{ Type of hernia } & 0.147 \\
\hline Unilateral & 45 & 0 & \\
\hline Bilateral & 322 & $15(4.5 \%)$ & \\
\hline \multicolumn{3}{|l|}{ Type of defect } & 0.368 \\
\hline Direct & 138 & $8(5.5 \%)$ & \\
\hline Indirect & 214 & $6(2.7 \%)$ & \\
\hline Combined & 15 & $1(6.3 \%)$ & \\
\hline \multicolumn{3}{|l|}{ Type of mesh } & 0.154 \\
\hline High weight & 323 & $15(4.4 \%)$ & \\
\hline Lightweight & 44 & 0 & \\
\hline \multicolumn{3}{|l|}{ Pore size } & 0.058 \\
\hline Micropore & 181 & $11(5.7 \%)$ & \\
\hline Macropore & 186 & $4(2.1 \%)$ & \\
\hline \multicolumn{3}{|l|}{ Mesh fixation } & 0.074 \\
\hline No & 66 & $3(4.3 \%)$ & \\
\hline Yes & 301 & $12(3.8 \%)$ & \\
\hline Titanium & 176 & $11(5.9 \%)$ & \\
\hline Absorbable & 125 & $1(0.8 \%)$ & \\
\hline Operative time $(\min \pm S D)$ & $39.8 \pm 14.2$ & $32.3 \pm 7.9$ & \\
\hline \multicolumn{3}{|l|}{ Learning curve } & 0.034 \\
\hline First 50 cases & 45 & $5(10 \%)$ & \\
\hline After 50 cases & 322 & $10(3.1 \%)$ & \\
\hline
\end{tabular}

Table 4. Multivariate analyses of the factors predicting pain and recurrence

\begin{tabular}{|l|l|l|l|}
\hline & Variable & OR $(\mathbf{9 5} \% \mathbf{C I})$ & $\mathbf{p}$ \\
\hline Pain & Pore size & $2.911(1.153-7.351)$ & 0.024 \\
\hline & $\begin{array}{l}\text { Mesh Fixation with } \\
\text { titanium tacks }\end{array}$ & $8.776(4.040-14.893)$ & 0.004 \\
\hline Recurrence & BMI & $1.307(1.138-1.501)$ & $<0.001$ \\
\hline \multicolumn{2}{|l|}{ OR: Odds ratio, Cl: Confidence interval, BMI: Body mass index } \\
\hline
\end{tabular}


pain, as defined before by The International Association for the Study of Pain. Our chronic pain rate was $8.1 \%$ and seemed to be higher than previous studies; however, the measurement tools and the definition of severe or chronic pain were quite different between our study and those others. Our mean VAS score at two years was compatible with the literature. The main drawback of our study may be the lack of quality of life questionnaires. Indeed, this is also a drawback of the literature that there are no standard definitions and measurement tools of postoperative pain and quality of life after inguinal hernia repair. There are few studies assessing the predictors of pain after TEP repair. Onlay mesh placement, fixation of the mesh, strenuous physical activity, young age, and poor general health have been suggested to increase postoperative chronic pain incidence (11-14). In our study, we found higher pain incidence with higher BMI, using microporous mesh, mesh fixation, fixation with titanium tacks, and at the beginning of the learning curve.

Risk factors for a recurrent inguinal hernia include female gender, direct versus indirect inguinal hernia, limited surgical experience, and low volume centers (7). Compatible with the literature, the recurrence rate was $10 \%$ at the beginning of our learning curve and decreased to 3.1\% after the first 50 cases $(p=0.034)$. Similarly, postoperative chronic pain was $14 \%$ and $7.2 \%$ before and after the $50^{\text {th }}$ case $(p=0.043)$. In a recent study, Berndsen et al. (14) reported a $2.5 \%$ recurrence after 485 laparoscopic inguinal hernia repairs at a 5-year followup. Strenuous work (OR: 13.7), technically challenging repairs (OR: 7.2), and chronic discomfort (OR: 6.7) were the risk factors for recurrence in their study. We did not assess the technical difficulty of the operation; however, operative time, which may be a predictor of difficulty, was associated with recurrence in our study $(p=0.040)$. Another drawback was the lack of records regarding postoperative activity and strenuous work of the patients; instead, we found higher BMI $(\mathrm{p}=0.008)$ and older age (0.034) were risk factors for recurrence.

There is an important heterogeneity among surgical technics and used materials, particularly in fixation fashion and mesh brands in TEP inguinal hernia repair. Recent guidelines strongly state that in almost all cases, any type of mesh fixation is unnecessary (7). Mesh fixation is recommended in only large direct hernias and with atraumatic fixation technics such as fibrin glue and cyanoacrylate $(7,15)$. Consistent with these recommendations, none of the patients with no-fixation in our study had chronic pain ( $0 \%$ vs $9.9 \%, p=0.002$ ). The recurrence rates were similar between no-fixation (4.3\%) and tack-fixation (3.8\%) groups $(p=0.074)$. Fixation with titanium tack was the only independent risk factor for chronic pain in our study. The main conflicting issue in laparoscopic TEP repair is selecting the best mesh material. Despite several published randomized trials and meta-analysis, there are no standardized recommendations. The porosity, elasticity, strength, and the material of the mesh all influence tissue reaction; however, a general classification, which is based on a specific property of the mesh and which can reflect all risks, is not currently available, and even hardly conceivable. The limited literature on those properties mostly focused on the weight classification (lightweight versus heavyweight), and no further details of the meshes were given in the published data. The Herniasurge group 2018 Guideline stated that there is no single perfect mesh, and a mesh classification reflecting all possible risks or benefits does not exist and is not possible to develop (7). Small pore and heavyweight meshes are considered to carry a high-risk for fibrotic bridging and long-term meshrelated problems. Only a few studies in the literature have compared lightweight and heavyweight meshes and shown the superiority of lightweight mesh regarding better pain scores, comfort, and sexual dysfunction over conventional heavyweight polypropylene (13). However, long-term results have raised concerns about an increased risk of mesh displacement and recurrence with the use of lightweight mesh $(16,17)$. A recent Swedish nationwide population study, including 13.839 laparoscopic TEP hernia repairs, revealed that lightweight meshes were associated with an increased risk of reoperation for recurrence (HR 1.56, $\mathrm{p}<0.001)$ compared to highweight meshes. This difference was more distinct in direct hernias and larger defects. In our study, there was no difference in chronic pain $(p=0.895)$ and recurrence $(p=0.368)$ rates between direct and indirect hernias. The chronic pain rates were also similar to lightweight (8\%) and highweight (9.1\%) meshes ( $p=0.490)$. There was no recurrence at two years with lightweight meshes, where in the highweight mesh group, our recurrence rate was $4.4 \%$, but this difference did not show a statistical significance $(p=0.154)$.

\section{CONCLUSION}

Laparoscopic TEP repair is a safe and effective method for inguinal hernia treatment. Mesh fixation is associated with a high risk of postoperative chronic pain with no benefit on longterm recurrence rates. Macroporous meshes provided favorable early postoperative outcome regarding pain. Mesh weight, pore size, and the brand did not affect recurrence. There is a need for large randomized trials in order to find the best classification of mesh materials and identify the perfect mesh. 


\section{Ethics}

Ethics Committee Approval: Approval of the local Ethics committee was received for the study (istanbul Medipol University Ethics committee number: 10840098-604.01.01E.53651).

Informed Consent: All the patients gave written consent for the surgery and permission for the use of data in studies.

Peer-review: Externally peer-reviewed.

\section{Authorship Contributions}

Surgical and Medical Practices: Y.Ö., P.B., Concept: Y.Ö., 0.0, Design: Y.Ö., N.C..A., Data Collection or Processing: P.B., O.O., Y.Ö., Analysis or Interpretation: N.Ç.A., Literature Search: N.C..A, Y.Ö., Writing: N.C..A., Y.Ö.

Conflict of Interest: No conflict of interest was declared by the authors.

Financial Disclosure: The authors declared that this study received no financial support.

\section{REFERENCES}

1. Bay-Nielsen M, Kehlet H, Strand L, Malmstrøm J, Andersen FH, Wara P, et al. Quality assessment of 26304 herniorrhaphies in Denmark: A prospective nationwide study. Lancet 2001;358:1124-8.

2. Hussain A, El-Hasani S. Chronic pain 5 years after randomized comparison of laparoscopic and Lichtenstein inguinal hernia repair $\mathrm{Br} J$ Surg 2010;97:600-608. Br J Surg 2010;97:1453. Author reply 1453-4.

3. Schmedt CG, Sauerland S, Bittner R. Comparison of endoscopic procedures vs Lichtenstein and other open mesh techniques for inguinal hernia repair: A meta-analysis of randomized controlled trials. Surg Endosc 2005;19:188-99.

4. Simons MP, Aufenacker T, Bay-Nielsen M, Bouillot JL, Campanelli G, Conze J, et al. European Hernia Society guidelines on the treatment of inguinal hernia in adult patients. Hernia 2009;13:343-403.

5. Chen LS, Chen WC, Kang YN, Wu CC, Tsai LW, Liu MZ. Effects of transabdominal preperitoneal and totally extraperitoneal inguinal hernia repair: an update systematic review and meta-analysis of randomized controlled trials. Surg Endosc 2018;33:418-28.
6. Köckerling F, Stechemesser B, Hukauf M, Kuthe A, Schug-Pass C. TEP versus Lichtenstein: Which technique is better for the repair of primary unilateral inguinal hernias in men? Surg Endosc 2016;30:3304-13.

7. HerniaSurge Group. International guidelines for groin hernia management. Hernia 2018;22:1-165.

8. Langeveld HR, Van'T Riet M, Weidema WF, Stassen LPS, Steyerberg EW, Lange J, et al. Total extraperitoneal inguinal hernia repair compared with lichtenstein (the level-trial): A randomized controlled trial. Ann Surg 2010;251:819-24

9. Westin L, Wollert S, Ljungdahl M, Sandblom G, Gunnarsson U, Dahlstrand U. Less pain 1 year after total extra-peritoneal repair compared with lichtenstein using local anesthesia data from a randomized controlled clinical trial. Ann Surg 2016;263:240-3.

10. Bansal VK, Misra MC, Babu D, Victor J, Kumar S, Sagar R, et al. A prospective, randomized comparison of long-term outcomes: Chronic groin pain and quality of life following totally extraperitoneal (TEP) and transabdominal preperitoneal (TAPP) laparoscopic inguinal hernia repair. Surg Endosc 2013;27:2373-82.

11. Patel LY, Lapin B, Gitelis ME, Brown C, Linn JG, Haggerty S, et al. Longterm patterns and predictors of pain following laparoscopic inguinal hernia repair: a patient-centered analysis. Surg Endosc 2017;31:210921.

12. Takata H, Matsutani T, Hagiwara N, Ueda J, Arai H, Yokoyama $\mathrm{Y}$, et al. Assessment of the incidence of chronic pain and discomfort after primary inguinal hernia repair. J Surg Res 2016;206:391-7.

13. Tolver MA, Rosenberg J, Bisgaard T. Convalescence after laparoscopic inguinal hernia repair: a qualitative systematic review. Surg Endosc 2016:30:5165-72.

14. Berndsen MR, Gudbjartsson T, Berndsen FH. Is a Technically Challenging Procedure More Likely to Fail? A Prospective Single-Center Study on the Short- and Long-Term Outcomes of Inguinal Hernia Repair. Surg Res Pract 2018;2018:7850671.

15. Buyukasik K, Ari A, Akce B, Tatar C, Segmen O, Bektas H. Comparison of mesh fixation and non-fixation in laparoscopic totally extraperitoneal inguinal hernia repair. Hernia 2017;21:543-8.

16. Agarwal BB, Agarwal KA, Mahajan KC. Prospective double-blind randomized controlled study comparing heavy- and lightweight polypropylene mesh in totally extraperitoneal repair of inguinal hernia: Early results. Surg Endosc 2009;23:242-7.

17. Chowbey PK, Garg N, Sharma A, Khullar R, Soni V, Baijal M, et al. Prospective randomized clinical trial comparing lightweight mesh and heavyweight polypropylene mesh in endoscopic totally extraperitoneal groin hernia repair. Surg Endosc 2010;24:3073-9. 